\title{
Exploring Teleimpedance and Tactile Feedback for Intuitive Control of the Pisa/IIT SoftHand
}

\author{
Arash Ajoudani, Student Member, IEEE, Sasha-B Godfrey, Member, IEEE, \\ Matteo Bianchi, Member, IEEE, Manuel Catalano, Member, IEEE, Giorgio Grioli, Member, IEEE, \\ Nikos Tsagarakis, Member, IEEE, and Antonio Bicchi, Fellow, IEEE
}

\begin{abstract}
-
This paper proposes a teleimpedance controller with tactile feedback for more intuitive control of the Pisa/IIT SoftHand. With the aim to realize a robust, efficient and low-cost hand prosthesis design, the SoftHand is developed based on the motor control principle of synergies, through which the immense complexity of the hand is simplified into distinct motor patterns. Due to the built-in flexibility of the hand joints, as the SoftHand grasps, it follows a synergistic path while allowing grasping of objects of various shapes using only a single motor. The DC motor of the hand incorporates a novel teleimpedance control in which the user's postural and stiffness synergy references are tracked in real-time. In addition, for intuitive control of the hand, two tactile interfaces are developed. The first interface (mechanotactile) exploits a disturbance observer which estimates the interaction forces in contact with the grasped object. Estimated interaction forces are then converted and applied to the upper arm of the user via a custom made pressure cuff. The second interface employs vibrotactile feedback based on surface irregularities and acceleration signals and is used to provide the user with information about the surface properties of the object as well as detection of object slippage while grasping. Grasp robustness and intuitiveness of hand control were evaluated in two sets of experiments. Results suggest that incorporating the aforementioned haptic feedback strategies, together with user-driven compliance of the hand, facilitate execution of safe and stable grasps, while suggesting that a low-cost, robust hand employing hardware-based synergies might be a good alternative to traditional myoelectric prostheses.
\end{abstract}

Index Terms-Prosthetic hand, Haptic feedback, Impedance control, Hand synergies, Under-actuation

\section{INTRODUCTION}

The primary causes of amputation are trauma, disease, and war, the proportions of which are skewed towards the former two in developed countries and the latter in the developing world. It is estimated that one in 200 people in the United States have a missing limb [1], although global numbers are difficult to estimate [2]. Following amputation, an individual must overcome significant physical and functional loss as well as the psychological trauma of change in independence and appearance. Prostheses have emerged to help cope with these immense changes. Body-powered prostheses were created to restore function and have seen great advancements in the last century. These prostheses provide several benefits including functionality, robustness, and limited sensory feedback. The next generation of prostheses has seen the incorporation of electronic components controlled via electromyographic (EMG) signals from the muscles in the residual limb. These

- Authors are with the Dept. of Advanced Robotics, Istituto Italiano di Tecnologia, Via Morego 30, 16163, Genoa, Italy. A. Ajoudani, M. Catalano, G. Grioli, and A. Bicchi are also with the Centro di Ricerca "E. Piaggio”, Universitá di Pisa, 56126 Pisa, Italy. E-mails: \{arash.ajoudani, nikos.tsagarakis, sasha.godfrey\}@iit.it, \{manuel.catalano, m.bianchi, giorgio.grioli, bicchi\}@centropiaggio.unipi.it. myoelectric prostheses can provide greater control of the hand and a more aesthetically pleasing appearance.

The most basic, and most common, myoelectric hands have a single degree of freedom (DOF) and incorporate various levels of control complexity. At a minimum, they offer on/off control and can be used by amputees with limited EMG signals. For users with more residual muscle and/or more control over those muscles, proportional controllers allow the user to vary grip force and/or grip speed relative to their EMG output, examples include the Hosmer myoelectric hand (Centri) or the DMC plus ${ }^{\circledR}$ (Otto Bock). In a technological leap forward, new anthropomorphic hands have emerged. The i-limb ${ }^{\mathrm{TM}}$ ultra (Touch Bionics, Inc) and the Bebionic 3 (RSL Steeper) are two of these that, while much more expensive than their basic counterparts, can adopt multiple realistic postures and grips. The trade-off of the increased DOFs and functionality is control complexity; the user must employ sequential contractions or co-contractions to select and operate the desired grip pattern.

Many different avenues of research are being pursued to improve on current commercial devices. One of these is employing complex machine learning algorithms to allow more natural control [3], [4]. This technique works well to classify a variety of postures in a lab setting, but depends on 
large training sets and few, if any, have been rigorously tested outside of the lab. To accommodate a larger functional range, the control burden on both the controller and the user necessarily grows. In response to this burden, the Pisa/IIT SoftHand [5] was developed as a joint venture between the Centro Piaggio of the University of Pisa and the Advanced Robotics Department of the Italian Institute of Technology. The SoftHand borrows from knowledge gained from neuroscience and motor learning to encode specific movement patterns, called synergies [6], [7], directly into the hand's hardware. The SoftHand actuates on the first synergy identified through Principal Component Analysis (PCA) of common hand movements, incorporating over $50 \%$ of the natural variety of the hand, and providing flexibility in the grasp pattern to facilitate molding around grasped objects.

To further exploit the efficiency and robustness of a synergybased hand, we developed a novel myoelectric teleimpedance controller. Teleimpedance control has previously replicated the impedance of the proximal arm [8], [9] and lower limb [10] and has proven itself a practical approach for real-time transfer of human impedance-regulation skills and equilibrium position profiles to robots. Here, we present an active impedance controller that incorporates both the hand stiffness and postural synergy references in real-time. Exploiting the concept of synergies that drive concurrent muscle activation, only one pair of antagonistic muscles (two EMG channels) was necessary for the musculoskeletal modeling of the grasp. The resulting model outputs then drove the stiffness and postural synergy references tracked by the developed impedance controller

\subsection{Haptics in Prosthesis: State of Art}

The hand is an especially complex part of the body containing a variety of sensing capabilities that can differentiate between light or firm touch, sense an object slipping from grasp, and determine the texture of an object, among others. Haptic information provided by mechanoreceptors of the fingertips is crucial to accomplish every day activities such as grip control and detection of object properties [11]. Further, recent research suggests feedback can aid in establishing a sense of embodiment or ownership of the prosthesis [12]. To the author's knowledge, no commercial devices provide the user with active feedback; however, the cable tension felt in bodypowered prostheses provides limited but functional feedback relating grip force to the user. To a lesser extent, motor noise provides auditory feedback to myoelectric users. Ultimately, prosthesis users must rely heavily on visual feedback to guide prosthetic use, thus limiting their usability. The research community is actively pursuing various methods of providing haptic feedback to prosthesis users to help mitigate this issue.

Feedback methods can be roughly broken down into two categories: invasive and non-invasive [13]. An example of the former is targeted reinnervation surgery, where severed nerves are rerouted from the amputated limb to residual muscles and skin [14]. The latter typically consists of delivering sensory feedback to intact sensory systems normally not involved in the task, e.g., on the forearm. Common haptic feedback modalities include vibrotactile feedback [13], skin stretch feedback
[15] and force feedback [16]. Such mechanical feedback was able to increase the acceptance and the ability to control the prosthetic hands [17]. Multi-modal haptic feedback is being actively pursued in research, such as [18], [19] and results suggest the most intuitive feedback is achieved through somatotopic matching (usually achievable via TR surgery) and modality matching, eg feeling pressure when exerting pressing on an object.

Below, we present the first steps towards adapting the Pisa/IIT SoftHand for prosthetic application and incorporating haptic feedback. This is done through two experiments: the first compares three different types of controllers (high and low fixed impedance against teleimpedance) both with and without force (low-frequency) feedback. To achieve this goal, we developed a grasp interaction force observer that relies on the pre-identified disturbance model of the hand. These interaction forces are then fed back to the user via the mechanical cuff, providing the user a sense of grasp forces in an intuitive manner. The second uses teleimpedance control and force feedback in conjunction with three levels of surface texture (high-frequency) feedback: no, moderate (2 finger), and full (4 finger) feedback. Since texture perception is important in activities of daily living [20], we developed a vibrotactile interface relying on surface irregularities and acceleration signals from the accelerometers mounted on the artificial hand, based on the works of [21], [22].

\section{THE PISA/IIT SOFTHAND}

The main design goal behind the development of the SoftHand [5] is the construction of a robust, safe, low-cost and, above all, simple robot hand. It is a well known fact in neuroscience that humans control their hands not merely by controlling each of its numerous degrees of freedom, but rather by coordinating and co-activating them in organized motions called synergies. Such behaviors have been numerically appraised in works such as [23] and [24]. In soft synergies, introduced in [7], the synergy serves as a reference position for a virtual hand, thus enabling better control of the interaction forces between the hand and the grasped object through variation of the virtual hand position or the stiffness matrix connecting the virtual and real hands. To manage weight, cost, and control complexity of the final device, underactuation [25], whereby fewer actuators are included than DOFs, was used in the design of the hand. This technique imparts a quality of shape adaptability to the device, thus these two strategies were combined to produce an "adaptive synergy" design strategy incorporating the neuroscientific basis of soft synergies with the shape adaptability of underactuation.

A second, but not less important, design goal pursued with the SoftHand was softness. By following the paradigms of soft robotics [26], [27] and [28] (chapters 13 and 57), the Pisa/IIT SoftHand is designed to be safe and robust, to make its use outside of strictly controlled lab environments a real possibility. An anthropomorphic hand was designed with 19 DOFs, 4 on each of 4 fingers, and 3 on the thumb. At rest, the hand measures roughly $23 \mathrm{~cm}$ from the tip of the thumb to the tip of the little finger, $23.5 \mathrm{~cm}$ from the wrist 
interface to the tip of the middle finger, and $4 \mathrm{~cm}$ thick at the palm. The fingers are capable of flexion/extension as well as ab/adduction. For ab/adduction of the fingers and at the equivalent of the carpometacarpal joint of the thumb (responsible for rotating the thumb from lateral pinch to $\mathrm{C}$ grasp, for example), traditional revolute joints were employed. The rest of the joints incorporate rolling contact joints with elastic ligaments, which ensure physiologically correct motions when actuated, but easily disengage on impact to allow safe interaction with humans while preserving the hand. The elastic ligaments also allow deformation while ensuring the hand returns to its original configuration. A single tendon runs though all joints to simultaneously flex and adduct the fingers upon actuation. The hand is actuated by a single DC motor which moves the fingers on the path of the first synergy as described in [23] allowing the SoftHand to mold around the desired object. The motor employed is a 6 Watt Maxon motor RE-max21 with an 84:1 gear reduction and a 12 bit magnetic encoder, resolution of $0.0875^{\circ}$ (Austrian Microsystems) and has a maximum holding torque of $2 \mathrm{Nm}$ and force of $20 \mathrm{~N}$ perpendicular to the palm.

\section{HUMAN-SOFTHAND INTERFACE}

Synergies are often thought of as kinematically coordinated movements of the hand joints [23]. Others give evidence to the existence of such pattern in dynamic coordinates [29], i.e., in the space of muscular activations [30], [31]. Aiming to use the minimum number of muscles necessary to decode and extract the hand stiffness and posture references, we used the major finger antagonist pair: the extensor digitorum communis (EDC) and flexor digitorum superficialis (FDS).

It is well known that muscle force increases with muscular activity [32]. While individual muscular forces affect and modify joint torques, their synchronized increase in antagonistic configuration actively adjusts the impedance of the corresponding joint [33]. By taking into account the forward dynamics of the grasp along the first synergy, we can write

$$
\begin{aligned}
\tau & =a_{\tau} \delta, \\
a_{\tau} \delta & =I \ddot{q}_{s}+c \dot{q}_{s}+K_{s}\left(q_{s}-q_{0}\right)+\tau_{E},
\end{aligned}
$$

where $\tau, a_{\tau}$ and $\tau_{E}$ denote the torque synergy, its gain and external torques, respectively; $q_{s}$ and $q_{0}$ are the position of the hand and the object along the first synergy; $\delta$ is a function of the difference in activation of the antagonistic muscles (FDS$E D C$ ), and $I, c$ and $K_{s}$ are the inertia, damping and stiffness of the hand along the first synergy, respectively. FDS and EDC are the processed EMG signals of the corresponding muscles. Now, by neglecting the effect of inertia and external torques in the above equation and rearranging it, we can write

$$
\dot{q}_{s}=\frac{-K_{s}}{c}\left(q_{s}-q_{0}\right)+\frac{a_{\tau}}{c} \delta
$$

The dynamics can be estimated in discrete time as follows

$$
q_{s_{k+1}}=\left(1-\frac{K_{s} T}{c}\right) q_{s_{k}}+\frac{T a_{\tau}}{c} \delta+\frac{K_{s} T}{c} q_{0}
$$

where $T$ and $k$ are the time step and iteration number. To establish $\delta$ and $K_{S}$ mappings based on experimental data, we used two functions of a modified hyperbolic tangent shape. The flexibility and capability of this function in the generation of various output profiles have been discussed in [34]. We write

$$
\delta=\frac{a_{q}\left[1-\mathrm{e}^{-b_{q}(\text { FDS }- \text { EDC })}\right]}{\left[1+\mathrm{e}^{-b_{q}(\text { FDS }- \text { EDC })}\right]},
$$

where $a_{q}$ and $b_{q}$ are the constant gains, to be identified experimentally. Similarly for the stiffness

$$
K_{s}=\frac{a_{k}\left[1-\mathrm{e}^{-b_{k}(\mathrm{FDS}+\mathrm{EDC})}\right]}{\left[1+\mathrm{e}^{-b_{k}(\mathrm{FDS}+\mathrm{EDC})}\right]},
$$

where $a_{k}$ and $b_{k}$ are similar as above and $K_{s}$ denotes the stiffness synergy reference, which is allocated in the stiffness interval of the robotic hand.

To identify the above parameters, FDS and EDC activity was recorded as subjects opened and closed the hand. As a reference, the SoftHand was slowly opened and closed. Subjects performed 20 grasping trials. Next, subjects were asked to perform the grasp at 5 different FDS and EDC cocontraction levels with visual feedback of co-contraction to assist in maintaining steady co-contraction levels. Four trials were recorded for each level, resulting in 40 trials total, 20 slow grasps and 20 grasps at various co-contraction levels.

Parameter identification of $\delta$ and stiffness model $\left(K_{S}\right)$, and the corresponding motor position reference and SoftHand stiffness level, respectively, was performed on even numbered trials while using FDS and EDC inputs. The odd numbered trials were used for the evaluation of the mappings. This led to the normalized root-mean-squared error (NRMSE) values of $17.6 \%$ and $13.4 \%$ for the postural and stiffness test trials, respectively, averaged across subjects. In the future, parameter identification of the models in amputees is likely feasible through standard training techniques such as mental imagery, teacher imitation, or bilateral action via mirror box [35].

\section{INTERACTION TORQUE OBSERVER}

As mentioned previously, a single DC motor is incorporated in the design of the SoftHand to pull the tendon and drive the finger joints according to the first hand synergy. The equation of motor dynamics ${ }^{1}$ is then defined by:

$$
J_{n} \ddot{q}=K_{t n} I_{r e f}-\tau_{d i s t},
$$

with $\ddot{q}, K_{t n}$, and $I_{r e f}$ denoting the motor angular acceleration, torque constant, and motor current, respectively. $J_{n}=J_{m}+$ $\frac{J_{h}}{N^{2}}$ represents the total inertia (motor inertia plus hand inertia reflected to the motor side).

In our setup, due to the low velocity profiles of the hand closure and the relatively high gear ratio, the reflected inertia of the hand, $\frac{J_{h}}{N^{2}}$, is neglected. The disturbance torque, $\tau_{d i s t}$, combines all the internal and external disturbance torques and is assumed to be formed by four components: the elastic torque generated by the hand tendons during closure $\left(\tau_{t e}\right)$, the gravitational effect $\left(\tau_{\text {grav }}\right)$, the frictional torque due to friction

1. In this paper, if not stated explicitly, all the variables and equations are described on the motor side (including interaction torque). Therefore, a gearbox ratio of $N=84$ must be taken into account for the presentation of the variables after the gearbox. 
in the hand joints and pulleys $\left(\tau_{f}\right)$, and the interaction torque $\left(\tau_{\text {int }}\right)$. We can write:

$$
\begin{aligned}
\tau_{\text {dist }} & =\tau_{\text {model }}+\tau_{\text {int }} \\
& =\tau_{\text {te }}+\tau_{f}+\tau_{\text {grav }}+\tau_{\text {int }} .
\end{aligned}
$$

Given the lightweight design of the hand, we can neglect $\tau_{\text {grav }}$ above. $\tau_{t e}$ is modeled as a function of the motor shaft rotation angle. In addition, the viscous and Coulomb friction of the hand is modeled using an antisymmetric piecewiselinear function of the motor speed and tendon tension [36], as follows,

$$
\tau_{f}(\dot{q})= \begin{cases}D_{1} \dot{q}+n_{s_{1}} K_{t e}\left(q-q_{o}\right) & \dot{q}>0 \\ D_{2} \dot{q}-n_{s_{2}} K_{t e}\left(q-q_{o}\right) & \dot{q}<0\end{cases}
$$

with $D_{i}, n_{s_{i}}, K_{t e}$, and $q_{o}$ representing the viscous damping and Coulomb friction coefficients, the reflected hand tendon stiffness, and motor angular position at rest (hand open), respectively. Incorporating the above assumptions in the disturbance model of the hand will result in:

$$
\tau_{\text {model }}= \begin{cases}\left(1+n_{s_{1}}\right) K_{t e}\left(q-q_{o}\right)+D_{1} \dot{q} & \dot{q}>0 \\ \left(1-n_{s_{2}}\right) K_{t e}\left(q-q_{o}\right)+D_{2} \dot{q} & \dot{q}<0,\end{cases}
$$

Therefore, the hand closure and opening models will be identified separately, as described below.

Supposing that the hand has not come in contact with the object to be grasped (i.e. $\tau_{i n t}=0$ in eq. 7 ), the hand model torque $\left(\tau_{\text {model }}\right)$ can be computed from the motor current and its motion response. Such calculation would require motor current and acceleration sensing with the latter being sensitive to noise if computed from position differentiation. To achieve reliable hand model torque estimation while taking into account the minimum hardware requirements, a robust torque observation technique is used here (Fig. 1). In particular, the hand model torque is estimated based on the angular velocity as follows:

$$
\begin{aligned}
\widehat{\tau}_{\text {model }} & =K_{\text {tn }} I_{\text {ref }}-J_{n} \ddot{q} \\
\widehat{\tau}_{\text {model }}(s) & \simeq \frac{\lambda}{s+\lambda}\left(K_{t n} I_{\text {ref }}-J_{n} s \dot{q}\right) \\
& \simeq \frac{\lambda}{s+\lambda}\left(K_{\text {tn }} I_{\text {ref }}+\lambda J_{n} \dot{q}\right)-\lambda J_{n} \dot{q} .
\end{aligned}
$$

Here, $s$ is the Laplace operator and $\lambda$ represents the filter cutoff frequency which affects the disturbance rejection capability [37]. The major design criterion is to choose $\lambda$ low enough to result in a robust system, while considering the introduced filtering delay. To estimate the reflected interaction torque caused by the contact of the hand with the environment, we take equations (6) and (10) into account and subtract the identified hand model torque (equation (9)), from the external torque effect as follows:

$$
\widehat{\tau}_{i n t}=\frac{\lambda}{s+\lambda}\left(K_{t n} I_{r e f}+\lambda J_{n} \dot{q}-\widehat{\tau}_{\text {model }}\right)-\lambda J_{n} \dot{q} .
$$

To identify the parameters of the hand model (equation 9), the hand controller was driven with fixed and low velocity (quasi-static) reference trajectories from the fully open to fully closed position. This process was repeated in the reverse direction as well, to account for the antisymmetric and velocity dependent properties of the friction model during opening and

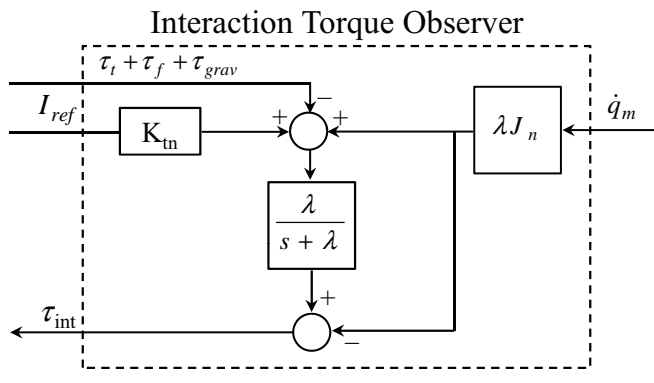

Fig. 1: Interaction torque observer block diagram.

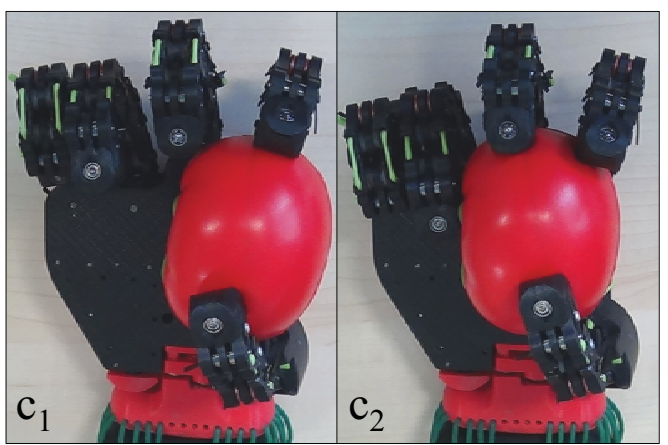

Fig. 2: The SoftHand molds around the obstacle with two $\left(\mathrm{c}_{1}\right)$ or three $\left(c_{2}\right)$ fingers during grasping.

closure. Next, the resultant current, position, and velocity profiles were used to estimate the components of equation (9), by means of conventional least squares identification algorithm. The identification process led to two feed-forward, velocity dependent estimates of the hand disturbance model, $\widehat{\tau}_{\text {model }}$. To evaluate our friction model, the hand stiffness parameter (outer position loop gain) was set to low values and a desired trajectory was tracked at different speeds through the full range of motion both with and without the presence of identified friction model. In each experiment, the mean absolute error (MAE) value for the motor position was calculated. In all cases, incorporation of the friction model resulted in more accurate tracking of the desired trajectory (e.g. \%35 reduction of MAE when the hand stiffness was set to $5 \mathrm{Nm} / \mathrm{rad}$ ), that gives evidence to the accuracy of the identified hand disturbance model.

In order to validate the accuracy of the identified hand model, the hand controller was provided with a sine wave position trajectory. The trajectory led to the execution of four SoftHand half-closure and full-opening movements. A soft, deformable ball was grasped during the second and third closures and removed on the first and the last. By predetermined placement of the soft obstacle along the hand closure, the hand conformed around the obstacle with two (Fig. $2-c_{1}$ ) or three fingers (Fig. 2-c $_{2}$ contacting the object, causing small deformations on the ball's surface. The motor current $\left(I_{\text {ext }}\right)$ was measured and used for the detection of contact and estimation of the interaction torque while grasping the ball.

Fig. (3) illustrates the results of this experiment. The top three plots demonstrate the position tracking, motor current, and motor voltage profiles, from the top down. Observed 
interaction torques once the soft obstacle is squeezed by two $\left(c_{1}\right)$ or three $\left(c_{2}\right)$ fingers are provided in the bottom plot. During the first and last closures, the fingers did not contact the object, resulting in low interaction torques. Although a soft and deformable object was grasped by only two or three fingers, interaction torque fluctuations were efficiently monitored.

\section{TACTILE INTERFACES}

The interaction that happens between the external environment and the user, mediated by a robotic or prosthetic hand, represents a typical tele-operation scenario, where the human user is the master and the artificial hand, exploring/grasping/manipulating external objects, is the slave. Under these conditions, in order to enable realistic perception, it is fundamental to provide the user with the haptic sensations arising from this interaction. More specifically, tool mediated activities comprise both low-frequency forces and highfrequency accelerations or vibrotactile stimuli. While it is straightforward to understand the need and the role of forcefeedback, e.g. to properly accomplish grasp operations and achieve stability, vibrotactile cues also convey essential haptic information, the absence of which might cause a haptic system to feel less natural [38].

\subsection{Mechano-tactile Feedback}

Mechanotactile displays have seen a large amount of progress, rendering relevant feedback stimuli to the human. Such tactile feedback is suggested to be beneficial for intuitive control of a prosthetic limb for transradial amputees and healthy users [39]. In our setup, a custom made, adjustable pressure cuff worn on the upper arm provided users with task interaction
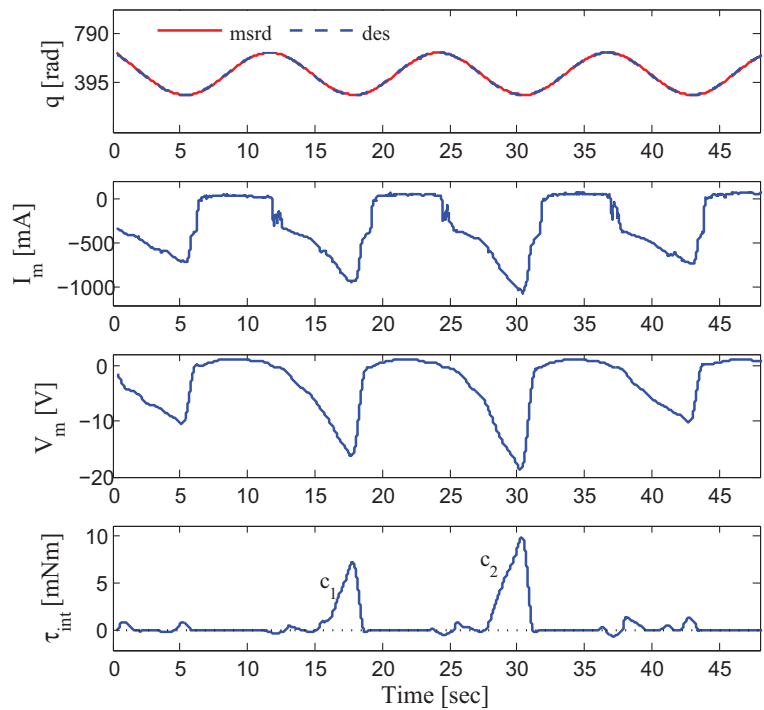

Fig. 3: Results of a grasping experiment with fixed stiffness gain. A soft, deformable ball was grasped during the second and third closures. The top three plots demonstrate the position tracking, motor current and motor voltage profiles, from the top down. Observed interaction torques once the soft obstacle was squeezed by two $\left(c_{1}\right)$ or three $\left(c_{2}\right)$ fingers are provided in the bottom plot.

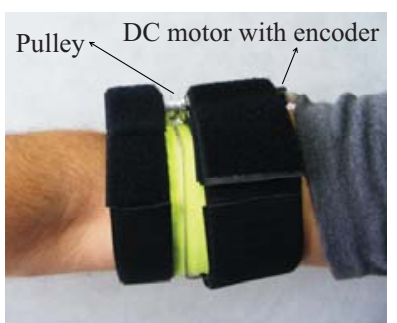

(a)

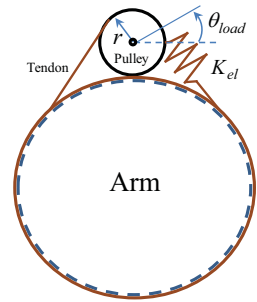

(b)
Fig. 4: Upper arm cuff, actuated by a DC motor picture (a) and loading scheme (b).

forces (see Fig. 4 and equation 11). A belt on the cuff is driven by a small DC motor to adjust the amount of applied mechanical pressure. The motor was driven by an active impedance controller, providing the possibility of establishing safe and reliable control of the applied pressure. The gains of the impedance controller were set experimentally and kept fixed among all experiments. The loading scheme of the upper arm cuff is shown in Fig. 4(b).

The estimated interaction torques (section 4) were converted, scaled, and tracked by the cuff's controller. To do so, the required amount of displacement for the cuff's motor $\left(\theta_{\text {load }}\right)$ was calculated based on the following equation

$$
\delta x_{\text {load }} \simeq \frac{a_{\text {cuff }} \tau_{\text {int }}}{K_{\text {el }}},
$$

where $a_{\text {cuff }}$ is an experimentally chosen coefficient that scales the observed interaction torques to a desired force to be applied with the cuff, varying between 0 and $14 \mathrm{~N}$, and $K_{e l}$ is the outer position gain of the cuff's controller (stiffness gain, chosen experimentally). $x_{\text {load }}$ is the tendon displacement ${ }^{2}$ and can be calculated from $r$ and $\theta_{\text {load }}$ in Fig. 4(b).

\subsection{Vibro-tactile Feedback}

Among surface properties, texture is one of the most important in everyday haptic perception [20], relying on surface irregularities and acceleration signals. In order to enhance the immersiveness of the haptic experience during tool-mediated and tele-operation tasks, it is, thus, crucial to replicate this high-frequency information arising from the contact of real objects and recorded on the tool (slave) side on the human (master) side. To achieve this goal, we developed an electronic acquisition and control circuit, following the scheme proposed in [21].

Acceleration data were recorded using an Analog Devices ADXL327 MEMS-based accelerometer, which is a small, low power, 3-axis accelerometer with signal conditioned voltage outputs and a minimum full-scale range of $\pm 2 \mathrm{~g}$. The sensors were mounted on the dorsal part of the distal phalanges of the five robotic fingers, chosen to minimize finger motion interference. We replicated on the master (human) side only

2. Here we assume that the cuff's tendon stiffness is very high and the damping effect is negligible due to the slow variations of the observed interaction torques. 
the $\mathrm{x}$-axis acceleration sensed by the robotic fingers, by means of vibrational feedback. The main motivations for this choice were: first, to maintain the simplicity of the acquisition/control circuit, in agreement with the synergy-driven design philosophy. Second, although we know that the complete acceleration signal depends on the sensing along all three axes, for our purposes the tangential $\mathrm{x}$-oriented acceleration contains the most useful information for dragging tasks and slipprevention applications. Third, trying to appropriately map multi-dimensional inputs to single axis actuators such as the ones we used introduces some technological problems. For a complete description of these problems as well as for a comparison and discussion of different solutions see [22]. Replicating only one axis acceleration from the slave to the master side represents a good trade-off between control simplicity and immersiveness of the haptic experience; it is also important to note that this information can be reproduced using a single axis actuator, despite its axis orientation, since the human hand can not discriminate vibration directions from one another [20].

As in [21], the accelerometer bandwidth is configured using an on-board first-order low-pass filter set at $1000 \mathrm{~Hz}$, followed by a DC block capacitor. Afterward, the signal is amplified with an adjustable gain $A$ (ranging from 0 to 4 ) and bandpass filtered. The band is from 46 to $307 \mathrm{~Hz}$ and is coherent with the one reported in [21]. It was chosen also to take into account the behavior of Pacinian corpuscles in human skin, which are sensitive to vibratory stimuli from 20 to $1000 \mathrm{~Hz}$, with a peak sensitivity between 250 and $550 \mathrm{~Hz}$ [40].

Finally the signal is applied to the motor, after current amplification (scaling factor up to 7), using a push-pull configuration. Four ${ }^{3}$ small $(24 \times 7 \mathrm{~mm})$, low-cost vibration motors (Precision Microdrives ${ }^{\mathrm{TM}}$ ) were sewn $15 \mathrm{~mm}$ apart on an adjustable-size cuff. The cuff was worn on the dorsal side of the forearm to avoid pressure on the vibrotactile motors from the forearm support. Fig. 5 demonstrates additional components of the SoftHand for the haptic experimental setup; the additional support/interface parts seen in the figure permit the attachment of the hand to non-amputee users for experimental validation of the system.

\subsection{Texture Rendering and Psychophysical Consid- erations}

The stimuli provided by the vibrotactile system are related to the texture of the surface explored by the robotic hand To be effective, such artificial feedback must be related to human perception capabilities and the physical roughness of the surface touched by the prosthetic hand. We took the vibrotactile detection thresholds and discriminating increments reported in [41] into consideration when designing our setup. Note also that vibration is strictly connected to roughness (texture) perception; indeed, vibration and hence the relative motion between skin and surface is the sine qua non for texture perception. In [42], the perception of roughness by active and passive touch was analyzed, revealing no difference between

3. To allow adequate spacing of the motors in the vibrotactile cuff, only digits $1-4$, thumb to ring finger, were used to provide texture feedback.

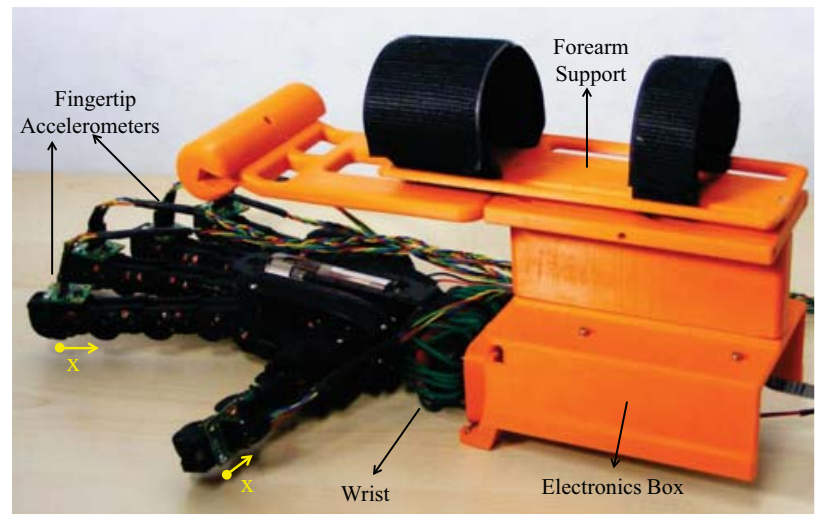

Fig. 5: SoftHand equipped with the able-bodied adapter (forearm support) and fingertip accelerometers.

the two haptic modalities. The results on vibration and texture perception as well as the findings on two-point discrimination reported in the previous subsection have been used to craft the proposed haptic system. To more closely approximate the surface roughness, the amplitude modulation of the vibromotor is directly related to the voltage provided by the control circuit and hence to the texture of the surface. To better clarify this point, we have performed some experiments using a KUKA/DLR Lightweight Robot arm with an ADXL327 MEMS-based accelerometer end-effector.

This accelerometer provided inputs to the control circuit that drives the motor. The Kuka arm was moved along the $\mathrm{y}$-axis (distance $13 \mathrm{~cm}$ ) with a linear trajectory and controlled in force in order to exert a maximum normal force along the $\mathrm{z}$-axis of $5 \mathrm{~N}$. The velocity was $5 \mathrm{~cm} / \mathrm{s}$ and the joint stiffness was set to $2000 \mathrm{~N} / \mathrm{mm}$ along the $y$ and $x$-axes and to $1 \mathrm{~N} / \mathrm{mm}$ along the $\mathrm{z}$-axis (perpendicular to the surface). Both force and velocity values were heuristically chosen to match typical parameters of the explorative actions performed by humans with the Pisa/IIT SoftHand. The Kuka was moved along two different surfaces: one smooth surface and one covered with sandpaper no. 40. While the end effector moved over each surface, the accelerometer recorded along the motion axis (yaxis). Meanwhile, the voltage output from the power stage of the circuit was measured and recorded with an oscilloscope. The Root Mean Square (RMS) of the voltage output for the sandpaper no. 40 was $0.8840 \mathrm{~V}$, while for the smooth surface it was $0.3859 \mathrm{~V}$. Interpolating from the specs of the motor, such values lead to motor vibration of $0.4119 \mathrm{~g}$ at $120 \mathrm{~Hz}$ and no vibration for the smooth case. The maximum operating voltage for the motor is $1.8 \mathrm{~V}$.

\section{Control Architecture}

As an alternative method to the bilateral master-slave teleoperation, teleimpedance control has been previously presented to overcome the stability issues raised in classical force-reflecting teleoperation scenarios, as well as enabling more natural task completion [9], [8]. In our proposed algorithm, task-oriented equilibrium position and stiffness profiles are estimated on the master side and realized by the teleoperated slave robot, in realtime. 


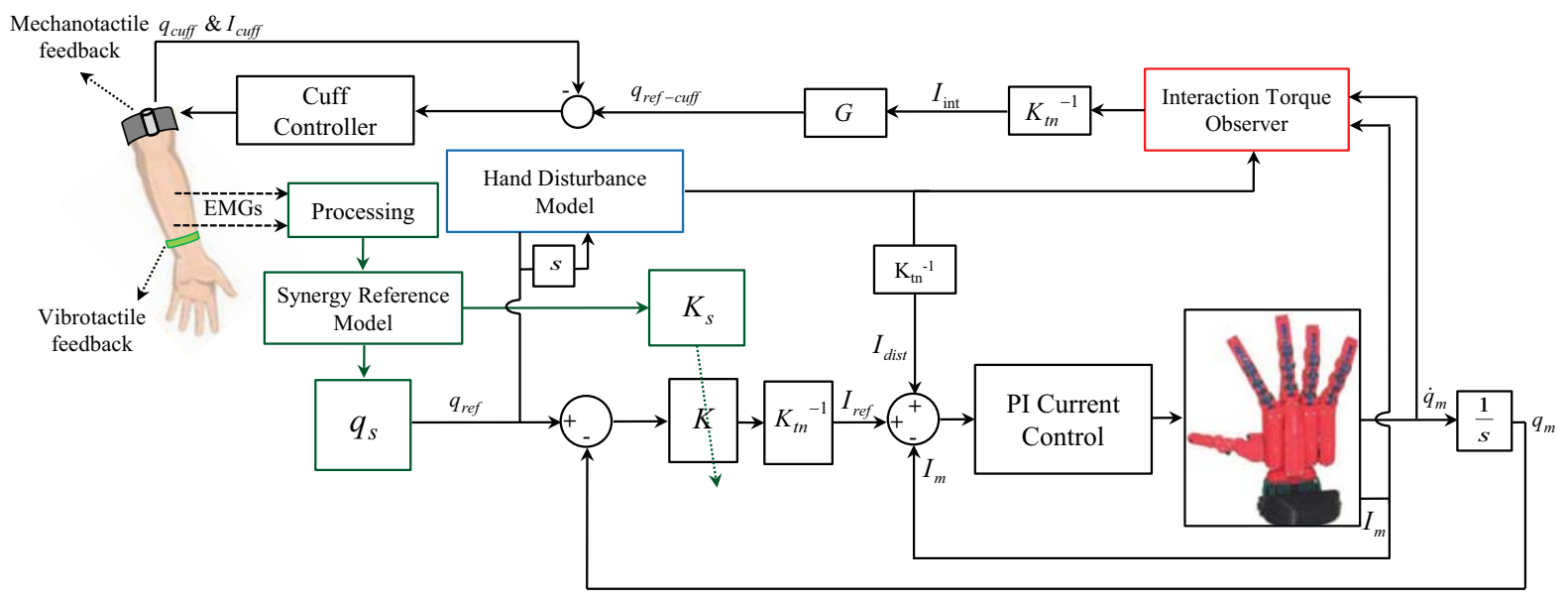

Fig. 6: Block diagram of the synergy-driven hand teleimpedance controller.

Here, to incorporate user intent into grasp control, a novel teleimpedance controller is developed. The block diagram of the proposed controller is shown in Fig. (6). The inner hand motor control loop is a high bandwidth current regulator while the outer loop implements an impedance controller, incorporating a time varying gain, and is updated by the user's hand stiffness synergy profile in realtime, based on the stiffness synergy model in section 3 . The bounds of the stiffness gain ([5 50] $\mathrm{Nm} / \mathrm{rad}$ ) were experimentally chosen to guarantee good tracking performance, stability, and high grasp compliance. Simultaneously, acquired muscular activities were used to determine reference position profiles as described earlier in section 3 .

The hand disturbance model block estimates the hand model torque, based on the feed-forward models (equation 9). The estimated torque is then converted to the current and fed to the inner current controller as $I_{d i s t}$. Simultaneously, the estimated hand model torque is used by the interaction torque observer (Fig. 1), to estimate the interaction torques due to contact with the grasped object. Subsequently, the resulting interaction torque is converted, scaled, and applied to the cuff's controller in order to provide the user with some indication of the grasp state and force (section 5.1).

\section{EXPERIMENTAL SETUP}

Analog electromyography signals were measured and amplified with a Delsys-Bangoli 16 (Delsys Inc.) apparatus. Acquired signals were band-pass filtered within the 20-450 $\mathrm{Hz}$ frequency range. Resulting signals were sampled at 2 $\mathrm{kHz}$ (PCI-6220, National Instruments) and full rectified for further processing. A digital, non-causal FIR linear phase lowpass filter was used for the detection of the envelope of the signal, which approximately corresponds to muscle activity. EMG normalization was performed automatically. Each time the system was activated, subjects were given 5 seconds to perform a large, brief co-contraction. This input was then used to normalize the EMG signals online. In addition, two online moving-average filters were implemented to detect the magnitude of drift in the lower bounds of each EMG channel, resulting from noise or change in limb configuration. The averaging was performed on a narrow and predefined amplitude interval of the normalized signals. At each time step, corresponding values were subtracted from the processed EMG measurements. Finally, resultant signals were fed into the model described in section 3 .

The hand unit and power driver for the motors (SoftHand and force feedback cuff) are custom control boards based on the Texas Instruments Luminary DSP chip LM3S8962. The DSP control loop is executed at $1 \mathrm{KHz}$ while the communication with the host PC is achieved through a real time Ethernet link. Motor current measurement is performed by a hall effect based current sensor (ACS714, Allegro Microsystems Inc.) and appropriate signal conditioning integrated in the motor power driver module. The data acquisition and synchronization interfaces between the motor controller boards, the interaction torque observer, the hand model torque, the EMG acquisition board, the hand synergy reference model, and the upper-arm cuff were developed in $\mathrm{C}++$. The acquisition, processing and control ran at $1 \mathrm{KHz}$ sampling frequency.

\subsection{Grasping Experiments}

The first experimental setup was designed to evaluate the effectiveness of fedback interaction forces and user-modified compliance in controlling the natural, robust grasp of the SoftHand. Objects with different elastic properties were grasped under the following hand controller parameters: i) fixed and relatively high stiffness gain (Stiff), ii) fixed and relatively low stiffness gain (Compliant), and iii) user modified hand compliance (Teleimpedance), all with or without the effect of interaction force feedback. Postural synergy commands were derived from the model, described in section 3, and were consistent among all experiments. Subjects were seated in front of a table and reached to grasp an object. Successful grasp was achieved when the SoftHand held the object securely off the surface of the table. Each grasp was attempted 3 times. To minimize any learning effects, subjects were provided with 
adequate training before the experiments and the order of the trials was randomized. Five right-handed subjects (one female, four male, mean age $=28.6$ ) participated in this setup.

\subsection{Haptic Experiments}

For the second experimental setup, five right-handed subjects (two female, three male, mean age=27) wore the SoftHand and the series of vibrotactile surface feedback motors on the forearm and the mechanotactile force feedback cuff on the upper arm (see Fig. 7). The setup for interacting with the test objects was modeled after the Haptic Black Box (HBB [43]) design. The objects were constrained within an experimental tray with low sides. A screen was placed parallel to this surface to occlude the objects from view without limiting the subject's visual feedback from the surrounding environment (to avoid balance issues, etc). Subjects were also provided with music via headphones to minimize the effect of auditory feedback which may impart texture information. Subjects were presented three pairs of objects before the experiment and then asked to retrieve the objects without visual or audio feedback. Each pair was formed by two objects of similar size, shape, and weight but with different surface properties. Object pairs were chosen to represent various typical shapes encountered and grasps used in activities of daily living. The first pair (Obvious) consisted of two similar plastic bars $(3 \times 18 \mathrm{~cm})$, one with a smooth surface and the other covered with a fine sand paper. An average-size apple and orange were used in the second (Mid) pair. And finally, the third pair (Subtle) was formed by two round, metal files $(25 \mathrm{~cm}$ long, with $1 \mathrm{~cm}$ file diameter, and $3 \mathrm{~cm}$ handle diameter) with either parallel or cross-cut teeth (see Fig. 7).

Each object pair was tested four times for each of three modes (36 trials for each subject): with mechanotactile feedback only (Mech), with Mech and partial (index and thumb) vibrotactile surface feedback (Half), and with Mech and full surface feedback (Full). For each object pair in each mode, subjects were asked to explore the space to find the desired object (two times for each object, in each object pair), and then grasp and lift it off the table. To minimize learning effects, subjects were allowed a familiarization period with each mode and the order of modes and object retrieval was randomized. Each trial was rated as successful or unsuccessful in two categories: choice, based on the selection of the correct object, and grasp, based on subjects ability to lift the object within three attempts.

In order to test for statistical significance of the results, the effects of the main factors (feedback modality and object pair) as well as the effects of their interaction must be analyzed. Since our data are binomial, and non-normally distributed, a classic two-way repeated measures ANOVA approach is not applicable; at the same time a Fisher-test for proportions can not be used since it does not take into account the within-subject vs. between-subject variability and the interaction effect between main factors. For these reasons, results were processed using Generalized Linear Mixed Models (GLMM), which are proven to be useful for the analysis of clustered/binomial data [44]. In GLMMs the overall variability consists of two different components: a fixed and a
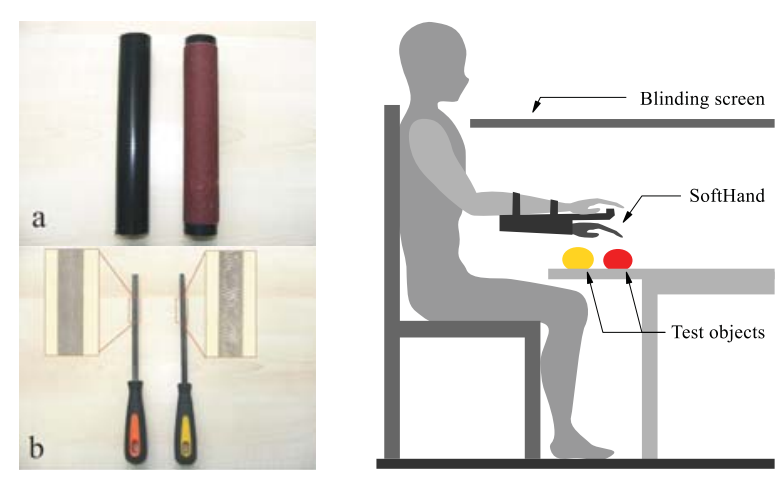

Fig. 7: At left, plastic bars (a) and the filing tools (b) used in haptic experiments; experimental setup at right.

random component. The fixed component takes into account and estimates the effects of interest, i.e. the experimental effects. In our case they are the feedback modality (Full, Half, Mech) and the object pair (Obvious, Mid, Subtle). The random component can estimate the heterogeneity between subjects. In this manner, a single model can be estimated across all subjects, but allowing each subject to have a different variability. Let $Y$ be the dependent variable, which is the dichotomous response variable (1 for a successful trial or 0 otherwise), whose values can be regarded as observations from the binomial distribution, with $i: 1 \ldots N$ subjects and $j=1 \ldots n_{i}$ repeated observations nested within each subject. In this case a classic mixed-effect logistic regression model can be used, whose formulation within the general GLMM framework can be expressed as

$$
P\left(Y_{i, j}=1 \mid v_{\mathbf{i}}, \mathbf{x}_{\mathbf{i j}}, \mathbf{z}_{\mathbf{i j}}\right)=g^{-1}\left(\eta_{i, j}\right)=\Psi\left(\eta_{i j}\right)
$$

where $\eta_{i j}$, the linear predictor, is given as: $\eta_{i j}=\mathbf{x}_{\mathbf{i}, \mathbf{j}}{ }^{\mathbf{T}} \beta+\mathbf{z}_{\mathbf{i j}}{ }^{\mathbf{T}} v_{\mathbf{i}}$. $\mathbf{x}_{\mathbf{i}, \mathbf{j}}$ is the vector of regressors, $\beta$ contains the fixed effect coefficients ${ }^{4}, \mathbf{z}_{\mathbf{i}, \mathbf{j}}$ is the vector of variables having random effects and $v_{\mathbf{i}}$ the vector of random effects. $g$ is the link function, which relates the response variables to the linear predictors. The logit function was used as the link function,

$$
g\left(\mu_{i j}\right)=\operatorname{logit}\left(\mu_{i j}\right)=\log \left[\frac{\mu_{i j}}{1-\mu_{i j}}\right]=\eta_{i j}
$$

where $\mu_{i j}=E\left(Y_{i j} \mid v_{\mathbf{i}}, \mathbf{x}_{\mathbf{i j}}\right)$ is the conditional expectation of the response variable, which equals $P\left(Y_{i j}=1 \mid v_{\mathbf{i}}, \mathbf{x}_{\mathbf{i j}}\right)$, i.e. the conditional probability of a response given the random effects. $\Psi\left(\eta_{i j}\right)$ is the inverse link function, in this case the logistic cumulative distribution function $(c d f)$.

\section{Results}

\subsection{Grasping Experiments}

As noted above, five subjects participated in grasping experiments. Subjects were able to grasp objects with different elastic properties with all controllers (see section 7 for details of the setup). Typical results of a trial grasping a rigid object (mug) are shown in Fig. 8 under Stiff (top), Compliant (mid), and Teleimpedance (bottom) controllers. The desired

\footnotetext{
4. Usually the intercept of the fit model is indicated by $\beta_{0}$.
} 


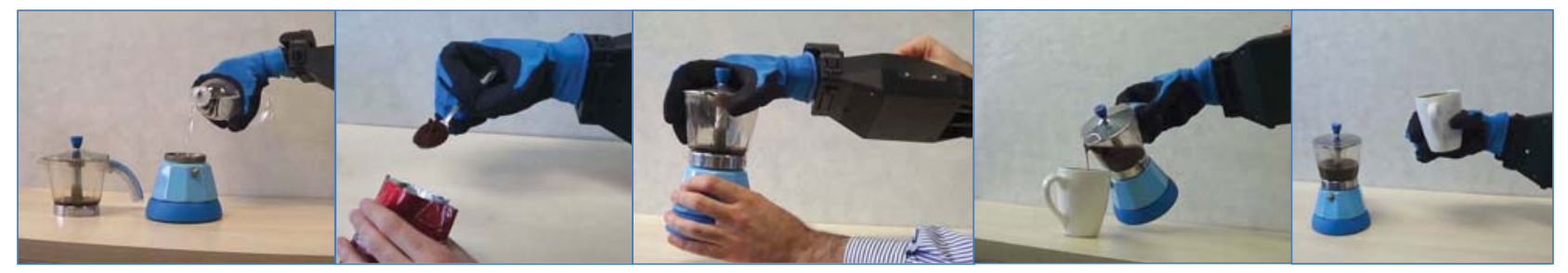

Fig. 9: SoftHand making an espresso.

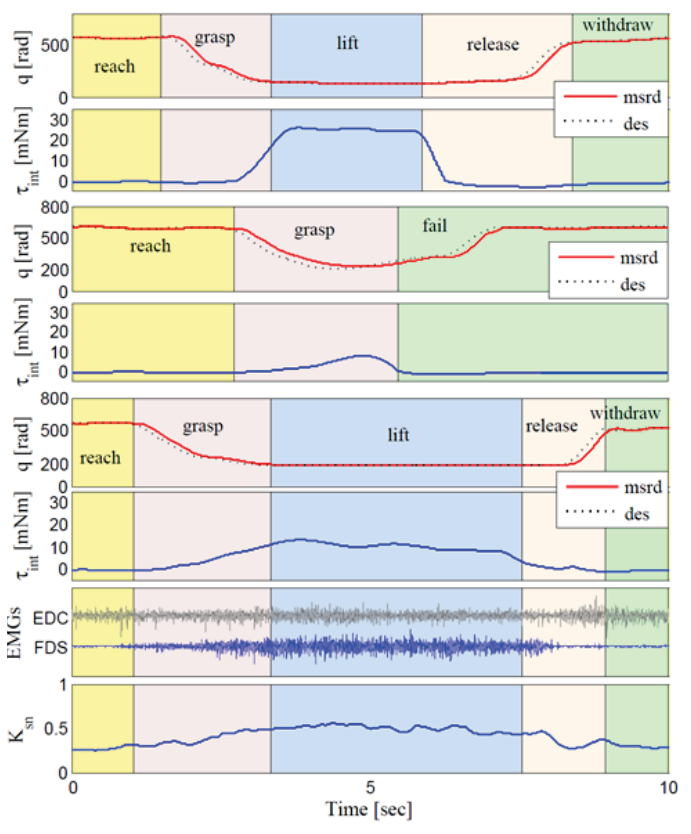

Fig. 8: Sample results of the SoftHand grasping a hard object (mug), with the controller under high, fixed stiffness gain (top pair, $K=40$ $\mathrm{Nm} / \mathrm{rad}$ ), low, fixed stiffness gain (mid pair, $K=10 \mathrm{Nm} / \mathrm{rad}$ ), and teleimpedance (bottom four, $a_{q_{n o r m}}=1, b_{q}=5.03, a_{k}=1.87$, and $b_{k}$ $=0.579$ ).

and measured postural synergy references and the observed interaction torques between the hand and the grasped object are shown for all controllers. Additionally, the subject's muscular activities and stiffness synergy reference, $K_{s}$ normalized to the chosen maximum stiffness gain $(50 \mathrm{Nm} / \mathrm{rad})$, while using the Teleimpedance controller are shown in the bottommost plot. As shown, the Stiff controller results in high interaction forces, which can cause damage or deformation to the object or prosthesis. In addition, abrupt changes in interaction forces are seen due to the rigidity of the hand. On the other hand, the Compliant controller with reduced stiffness gain produced lower interaction forces but was unable to provide the grasp forces required to complete the task. Unlike the Compliant and Stiff cases, user-modified compliance of the hand used in Teleimpedance control, together with the postural synergy profiles, provided the possibility of adjusting task-related grasp forces. Similar results were seen across subjects and trials: the Compliant controller had a higher failure rate than the other modes despite fairly good position tracking, while the Stiff controller more frequently caused undesired deformations on the object's surface. In contrast, with Teleimpedance, lower co-contractions resulted in high compliance, allowing gentle grasping of fragile or deformable objects, while higher stiffness values were generated with higher co-contractions to grasp heavier or more rigid objects. Fig. 10.a shows the average interaction forces across subjects and objects grasped with each controller, further illustrating the above. Fig. 10.b shows the effect of feedback on co-contraction (FDS + EDC) levels with Teleimpedance. The SoftHand teleimpedance controller was also tested with activities of daily living (e.g. opening and closing a jar lid, etc). In Fig. (9), a subject uses the SoftHand to demonstrate the steps to make an espresso.

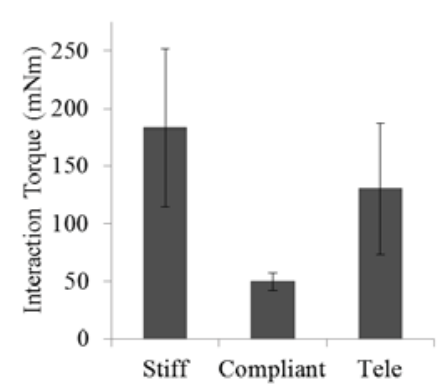

(a)

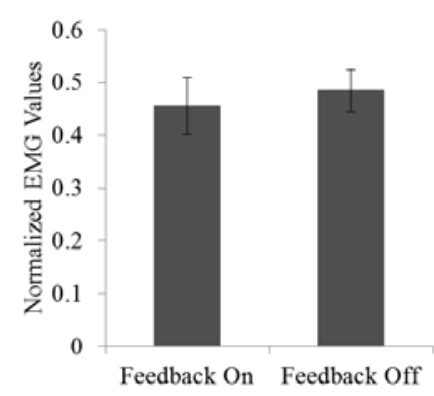

(b)
Fig. 10: a) Average interaction forces for five subjects grasping different objects with SoftHand controlled under Stiff, Compliant and Teleimpedance controllers. b) Average processed EMG signals for five subjects, grasping different objects. The averaging was performed on all trials either with or without the effect of interaction force feedback in Teleimpedance experiments.

\subsection{Haptic Experiments}

As described in the Experimental Setup section above, we used a GLMM to analyze the Haptic Experiment results. Let $Y$, the dichotomous response variable, be the dependent variable. The explanatory variables for the fixed component we used as regressors (in $\mathbf{x}_{\mathbf{i j}}$ in eq. (13)) are the two main factors we want to investigate: feedback modality $(F d)$, with levels Full, Half, and Mech, and object pair $(O b)$, with levels Obvious, Mid, and Subtle. The random effect (modeled in $\mathbf{z}_{\mathbf{i j}}$ in eq. (13)) takes into account the heterogeneity between subjects, for example the variance in distribution of response. The model assumes a single random effect (random intercept), which allows each participant to have a different intercept. The single random effect is assumed to have a Gaussian distribution with zero mean and variance estimated from the data (fitted variance equal to $1.628 \times 10^{-10}$ and 0.4629 for 
the haptic data and grasp data, respectively). GLMM models were fit to grasp and haptic data: (1) in the first model $(F d * O b)$ both the main effects and their interaction were taken into account; (2) in the second model $(F d+O b)$, only main effects without interaction were considered. (For a complete discussion on fitting GLMMs, the reader is referred to [45]. A maximum likelihood (ML) estimation was used to find the coefficients of the model, solved numerically using the GaussHermite quadrature implemented in the function glmer in $\mathbf{R}$ package $l m e 4^{5}$. The Likelihood Ratio test (LR) and the Akaike Information Criterion (AIC) [44] are common procedures for model comparison. (Note: lower AIC values indicate better fit.) Likelihood Ratio test (LR) reveals no significant difference $(p>0.4)$ between the fit of the two models 1 and 2 described above. This means that the interaction term between the two effects of interest does not add any significant factor for model fitting to the data, hence it can be disregarded. Furthermore, model $(F d+O b)$ exhibits the smallest value for the Akaike Information Criterion (AIC), for both grasp and haptic data. These observations lead us to use model $F d+O b$ and to disregard any interaction between the two main factors. The following tables contain the results of data fitting, where fitting coefficients $(\beta)$ for the fixed effects are listed along with their significance assessed by Wald statistics. Note that the fitting coefficients for the fixed effects provide contrasts between the indicated level and the omitted reference level. For both types of data, we considered two pairs of reference levels for a total of four tests: Full and Obvious (Tables 1 and 3, for haptic and grasp data, respectively) and Half and Mid (Tables 2 and 4, for haptic and grasp data, respectively).

We adopted Bonferroni post-hoc correction for multiple comparisons. For the haptic data, results from Table 1 suggest a trend towards a positive effect of Full compared to Mech, although this does not reach significance after post-hoc correction. A possible conclusion that can be drawn from the experimental outcomes is that the full vibrotactile feedback compared with the purely mechanical one seems to provide important additional information for haptic discrimination. For Object Pair, a significant positive effect of Obvious w.r.t. Mid was observed $(p=0.002)$. A trend towards a positive effect was also observed for Subtle vs. Obvious and Subtle vs. Mid that are not significant after post-hoc correction. These results might indicate a difficulty in the discrimination for the subtle object pair, which might be solved by increasing the training period for participants during the experiments while they manipulate different object pairs. For grasp data, a positive, significant effect of Full compared to Mech was observed ( $p=0.001)$, suggesting a significant effect of the full vibrotactile feedback compared with the purely mechanical one for grasp actions. For object pairs, a significant positive effect of Obvious w.r.t Mid and Obvious w.r.t Subtle was observed ( $p=0.03$ and $p=0.001$, respectively). These results suggest that full vibrotactile feedback seems to enhance both haptic discrimination and grasp actions and that object pairing also plays a significant role in both of these.

5. The R Project for Statistical Computing - www.r-project.org; package "Ime4: Linear mixed-effects models using s4 classes. $\mathrm{r}$ package version 0.999375-39” 2011. D. Bates, M. Maechler, and B. Bolker
TABLE 1: Results of the fit of $F d+O b$ applied to haptic discrimination data using Full and Obvious reference levels. In the first column the Coefficients are reported, then their estimate and standard (Std) error. The $\mathrm{Z}$ values refer to the standard normal distribution $\mathrm{Z}$ used to get the p-values reported in the last column (Wald statistics). Signif. codes: $0 * * *, 0.001 * *, 0.01 *, 0.05 ., 0.1,1$.

\begin{tabular}{cccccc}
\hline \hline Coefficients & Estimate & Std. Error & $\mathrm{z}$ value & $\operatorname{Pr}(>|z|)$ & \\
\hline (Intercept) & 4.0454 & 0.8345 & 4.848 & $1.25 \mathrm{e}-06$ & $* * *$ \\
Half & -0.6394 & 0.5757 & -1.111 & 0.266755 & \\
Mech & -1.1153 & 0.5544 & -2.012 & 0.044266 & $*$ \\
Mid & -2.6524 & 0.7767 & -3.415 & 0.000638 & $* * *$ \\
Subtle & -1.6544 & 0.8091 & -2.045 & 0.040873 & $*$ \\
\hline
\end{tabular}

TABLE 2: Similar to Table 1. Results of haptic data fit with $F d+O b$ using Half and Mid reference levels.

\begin{tabular}{cccccc}
\hline \hline Coefficients & Estimate Std. & Error & $\mathrm{z}$ value & $\operatorname{Pr}(>|z|)$ & \\
\hline (Intercept) & 0.7536 & 0.4080 & 1.847 & 0.064774 &. \\
Full & 0.6394 & 0.5757 & 1.111 & 0.266758 & \\
Mech & -0.4759 & 0.4917 & -0.968 & 0.333123 & \\
Obvious & 2.6524 & 0.7767 & 3.415 & 0.000638 & $* * *$ \\
Subtle & 0.9980 & 0.4639 & 2.151 & 0.031448 & $*$ \\
\hline
\end{tabular}

\section{Discussion}

A novel synergy-based teleimpedance controller was developed to gather the user's postural and stiffness synergy references from two EMG channels. Two nonlinear functions were used to map the EMGs to the postural and stiffness synergies. Resulting synergy commands were then tracked by the developed SoftHand controller in realtime. In addition, two haptic interfaces (Mechano- and vibrotactile) were developed to facilitate natural control of the prosthetic limb. The mechanotactile interface provided the user with information on grasp forces, to enable intuitive modulations of task-required forces, while the vibrotactile interface provided the user with information about object slippage and perception of surface properties such as texture.

The teleimpedance controller, both with and without haptic interfaces, was evaluated through two sets of experiments. The first set was designed to assess the capabilities of the teleimpedance controller with force feedback in natural grasping. Modification of grasp compliance via Teleimpedance controller resulted in robust and reliable grasps, regardless of the elastic properties of the grasped object. This study showed a slight but not significant reduction of physiological load with force feedback, possibly because of the limited training time and/or the small number of subjects used in this pilot study. Future work will expand these results and tease out the effects of the mechanotactile force feedback.

In the second set of experiments, force feedback and the teleimpedance controller were used in combination with vibrotactile texture feedback for the detection and grasping of objects with different surface properties. The results suggest the full vibrotactile feedback modality seems to enhance both haptic discrimination and grasp ability. However, full vibrotactile feedback appears to play a more dominant and significant role in grasp tasks than in haptic discrimination tasks. Object pair also played a role in both haptic discrimination, as expected, and grasp ability, with the large spheres being 
TABLE 3: Similar to Table 1. Results of grasp data fit with $F d+O b$ using Full and Obvious reference levels.

\begin{tabular}{cccccc}
\hline \hline Coefficients & Estimate Std. & Error & $\mathrm{z}$ value & $\operatorname{Pr}(>|z|)$ & \\
\hline (Intercept) & 2.6804 & 1.2114 & 4.633 & $3.61 \mathrm{e}-06$ & $* * *$ \\
Half & -0.7647 & 0.6335 & -1.207 & 0.227379 & \\
Mech & -2.1214 & 0.6021 & -3.523 & 0.000427 & $* * *$ \\
Mid & -2.9315 & 1.1052 & -2.653 & 0.007990 & $* *$ \\
Subtle & -3.8765 & 1.0983 & -3.530 & 0.000416 & $* * *$ \\
\hline
\end{tabular}

TABLE 4: Similar to Table 1. Results of grasp data fit with $F d+O b$ using Half and Mid reference levels.

\begin{tabular}{cccccc}
\hline \hline Coefficients & Estimate Std. & Error & $\mathrm{z}$ value & $\operatorname{Pr}(>|z|)$ & \\
\hline (Intercept) & 1.9158 & 0.5808 & 3.299 & 0.000972 & $* * *$ \\
Full & 0.7647 & 0.6335 & 1.207 & 0.227366 & \\
Mech & -1.3567 & 0.5163 & -2.628 & 0.008598 & $* *$ \\
Obvious & 2.9316 & 1.1052 & 2.653 & 0.007989 & $* *$ \\
Subtle & -0.9450 & 0.4678 & -2.020 & 0.043360 & $*$ \\
\hline
\end{tabular}

most difficult to grasp successfully. Although these results are preliminary and should be validated with a larger number of subjects and trials, they illustrate the feasibility of effective and simple haptic interfaces to control artificial robotic hands in common actions.

The mechanotactile-only results suggest meaningful texture information transfers through the device and forearm support to inform the user. However, additional feedback may provide added benefit, as seen with the full- and half-haptic results, especially in amputees where socket liners provide additional damping. The additional haptic feedback also provided benefit in blind grasping, a feature potentially useful to amputees given the current reliance on visual feedback to execute successful grasps. With respect to grasp success by object shape, it is worth noting the ease with which users grasped the cylinder and the high failure rate and variability for the sphere. Anecdotally, the SoftHand was able to mold around the cylinder from a myriad of starting positions. In comparison, subjects would misalign the SoftHand with the sphere, resulting in object slippage before lift off or orient the SoftHand palm at the top of the sphere, resulting in the fingers failing to reach the sphere. This phenomenon is likely also attributable to the large size of the spheres tested; smaller and/or more compliant spheres would lend themselves to a wider variety of grasping strategies and likely have higher grasp success rates. Grasping of the sphere was best with full-haptic feedback, likely because users were better able to understand the position of the SoftHand relative to sphere.

In the future, further investigation of optimal stimulation sites and spacing for various feedback modalities will be examined and this investigation will be extended to amputees. While these results need to be validated with amputees, they provide preliminary evidence that a low-cost, robust hand employing hardware-based synergies might be a good alternative to traditional myoelectric prostheses. Furthermore, they illustrate the benefits of incorporating tactile interfaces for more intuitive control of the SoftHand.

\section{APPENDIX A}

\section{ACKNOWLEDGMENTS}

This work is supported in part by the European Research Council under the Advanced Grant SoftHands "A Theory of Soft Synergies for a New Generation of Artificial Hands" no. ERC-291166, and by the EU FP7 project (601165), "WEARable HAPtics for Humans and Robots (WEARHAP)".

\section{REFERENCES}

[1] N. Staff, "Amputation statistics by cause, limb loss in the united states," National Limb Loss Information Center, Knoxville, TN, 2008.

[2] World Health Organization, United States Department of Defense, and MossRehab Amputee Rehabilitation Program, "The rehabilitation of people with amputations," World Health Organization, 2004.

[3] N. Jiang, K. Englehart, and P. Parker, "Extracting simultaneous and proportional neural control information for multiple-dof prostheses from the surface electromyographic signal," Biomedical Engineering, IEEE Transactions on, vol. 56, no. 4, pp. 1070-1080, 2009.

[4] D. Naidu, C. Chen, A. Perez, and M. Schoen, "Control strategies for smart prosthetic hand technology: An overview," in Engineering in Medicine and Biology Society, 2008. EMBS 2008. 30th Annual International Conference of the IEEE. IEEE, 2008, pp. 4314-4317.

[5] M. G. Catalano, G. Grioli, A. Serio, E. Farnioli, C. Piazza, and A. Bicchi, "Adaptive synergies for a humanoid robot hand," in IEEERAS International Conference on Humanoid Robots, Osaka, Japan, In Press.

[6] M. Gabiccini, A. Bicchi, D. Prattichizzo, and M. Malvezzi, "On the role of hand synergies in the optimal choice of grasping forces," Autonomous Robots, vol. 31, no. 2, pp. 235-252, 2011.

[7] A. Bicchi, M. Gabiccini, and M. Santello, "Modelling natural and artificial hands with synergies," Philosophical Transactions of the Royal Society B: Biological Sciences, vol. 366, no. 1581, pp. 3153-3161, 2011.

[8] A. Ajoudani, N. Tsagarakis, and A. Bicchi, "Tele-impedance: Towards transferring human impedance regulation skills to robots," in International Conference of Robotics and Automation - ICRA 2012, Saint Paul, MN, USA, May 14 - 18 2012, http://www.youtube.com/watch?v=Fn2dObnFpw.

[9] A. Ajoudani, N. G. Tsagarakis, and A. Bicchi, "Tele-Impedance: Teleoperation with impedance regulation using a body-machine interface," International Journal of Robotics Research, vol. 31(13), pp. 1642-1655, 2012, http://www.youtube.com/watch?v=KPO6IO7Tr-Q.

[10] N. Karavas, A. Ajoudani, N. G. Tsagarakis, J. Saglia, A. Bicchi, and D. G. Caldwell, "Tele-impedance based stiffness and motion augmentation for a knee exoskeleton device," in IEEE International Conference on Robotics and Automation (ICRA2013), In Press.

[11] G. Cadoret and A. Smith, "Friction, not texture, dictates grip forces used during object manipulation,” J. Neurophysiol., vol. 75, pp. 1963-1969, 1996.

[12] P. D. Marasco, K. Kim, J. E. Colgate, M. A. Peshkin, and T. A Kuiken, "Robotic touch shifts perception of embodiment to a prosthesis in targeted reinnervation amputees," Brain, vol. 134, no. 3, pp. 747-758, 2011.

[13] C. Cipriani, M. D’Alonzo, and M. Carrozza, "A miniature vibrotactile sensory substitution device for multi-fingered hand prosthetics," IEEE TBME, 2011.

[14] T. Kuiken, L. Miller, R. Lipschutz, B. Lock, K. S. eld, P. Marasco, P. Zhou, and G. Dumanian, "The use of targeted muscle reinnervation for improved myoelectric prosthesis control in a bilateral shoulder disarticulation amputee," Prosthetics Orthotics Int., vol. 28, no. 3, pp. 245-253, 2004.

[15] J. Wheeler, K. Bark, J. Savall, and M. Cutkosky, "Investigation of rotational skin stretch for proprioceptive feedback with application to myoelectric systems," IEEE Transactions on Neural Systems and Rehabilitation Engineering, vol. 18, no. 1, pp. 58-66, 2011.

[16] S. Meek, S. Jacobsen, and P. Goulding, "Extended physiologic taction: Design and evaluation of a proportional force feedback," J. Rehabil. Res. Dev., vol. 26, no. 3, pp. 53-62, 1989.

[17] J. Brown, A. Paek, M. Syed, M. O’Malley, P. Shewokis, J. ContrerasVidalk, A. Davis, and R. Gillespie, "Understanding the role of haptic feedback in a teleoperated/prosthetic grasp and lift task," in 2013 IEEE World Haptics Conference (WHC), 2013, pp. 271-276. 
[18] K. Kim and J. Colgate, "Haptic feedback enhances grip force control of semg-controlled prosthetic hands in targeted reinnervation amputees," Neural Systems and Rehabilitation Engineering, IEEE Transactions on, vol. 20, no. 6, pp. 798-805, 2012.

[19] M. D'Alonzo, S. Dosen, C. Cipriani, and D. Farina, "Hyve-hybrid vibro-electrotactile stimulation-is an efficient approach to multi-channel sensory feedback," Transactions on Haptics, 2013.

[20] N. Landin, J. Romano, W. McMahan, and K. Kuchenbecker, "Dimensional reduction of high-frequency accelerations for haptic rendering," in Haptics: Generating and Perceiving Tangible Sensations, ser. Lecture Notes in Computer Science, A. Kappers, J. Erp, W. Bergmann Tiest, and F. Helm, Eds. Springer Berlin Heidelberg, 2010, vol. 6192, pp. 79-86.

[21] K. Kuchenbecker, J. Gewirtz, W. McMahan, D. Standish, P. Martin, J. Bohren, P. Mendoza, and D. Lee, "Verrotouch: High-frequency acceleration feedback for telerobotic surgery," in Haptics: Generating and Perceiving Tangible Sensations, ser. Lecture Notes in Computer Science, A. Kappers, J. Erp, W. Bergmann Tiest, and F. Helm, Eds. Springer Berlin Heidelberg, 2010, vol. 6191, pp. 189-196.

[22] J. Romano and K. Kuchenbecker, "Creating realistic virtual textures from contact acceleration data," Haptics, IEEE Transactions on, vol. 5, no. 2, pp. 109-119, 2012.

[23] M. Santello, M. Flanders, and J. Soechting, "Postural hand synergies for tool use," The Journal of Neuroscience, vol. 18, no. 23, pp. 10105 10115,1998

[24] P. H. Thakur, A. J. Bastian, and S. S. Hsiao, "Multidigit movement synergies of the human hand in an unconstrained haptic exploration task," The Journal of neuroscience, vol. 28, no. 6, pp. 1271-1281, 2008.

[25] L. Birglen, C. Gosselin, and T. Laliberté, Underactuated robotic hands. Springer Verlag, 2008, vol. 40.

[26] A. Albu-Schaffer, O. Eiberger, M. Grebenstein, S. Haddadin, C. Ott, T. Wimbock, S. Wolf, and G. Hirzinger, "Soft robotics," Robotics \& Automation Magazine, IEEE, vol. 15, no. 3, pp. 20-30, 2008

[27] D. Trivedi, C. D. Rahn, W. M. Kier, and I. D. Walker, "Soft robotics: Biological inspiration, state of the art, and future research," Applied Bionics and Biomechanics, vol. 5, no. 3, pp. 99-117, 2008.

[28] B. Siciliano and O. Khatib, Springer handbook of robotics. Springer, 2008 .

[29] V. M. Zatsiorsky and M. L. Latash, "Multifinger prehension: an overview," Journal of Motor Behavior, vol. 40, no. 5, pp. 446-476, 2008.

[30] W. Lee, "Neuromotor synergies as a basis for coordinated intentional action," Journal of Motor Behavior, vol. 16, no. 2, pp. 135-170, 1984.

[31] M. Flanders and J. F. Soechting, "Arm muscle activation for static forces in three-dimensional space," Journal of Neurophysiology, vol. 64, no. 6, pp. $1818-1837,1990$.

[32] H. Milner-Brown and R. Stein, "The relation between the surface electromyogram and muscular force." The Journal of physiology, vol. 246, no. 3, pp. 549-569, 1975 .

[33] R. Osu and H. Gomi, "Multijoint muscle regulation mechanisms examined by measured human arm stiffness and emg signals," Journal of neurophysiology, vol. 81, no. 4, pp. 1458-1468, 1999.

[34] C.-T. Chen and W.-D. Chang, "A feedforward neural network with function shape autotuning," Neural networks, vol. 9, no. 4, pp. 627641,1996

[35] C. Castellini, E. Gruppioni, A. Davalli, and G. Sandini, "Fine detection of grasp force and posture by amputees via surface electromyography," Journal of Physiology-Elsevier, vol. 103, no. 3, p. 255, 2009.

[36] C. C. De Wit and P. Lischinsky, "Adaptive friction compensation with partially known dynamic friction model," International journal of adaptive control and signal processing, vol. 11, no. 1, pp. 65-80, 1997.

[37] T. Murakami, F. Yu, and K. Ohnishi, "Torque sensorless control in multidegree-of-freedom manipulator," Industrial Electronics, IEEE Transactions on, vol. 40, no. 2, pp. 259-265, 1993.

[38] W. McMahan, J. Romano, A. Abdul Rahuman, and K. Kuchenbecker "High frequency acceleration feedback significantly increases the realism of haptically rendered textured surfaces," in Haptics Symposium, 2010 IEEE, 2010, pp. 141-148.

[39] C. Antfolk, M. D’Alonzo, M. Controzzi, G. Lundborg, B. Rosen, F. Sebelius, and C. Cipriani, "Artificial redirection of sensation from prosthetic fingers to the phantom hand map on transradial amputees: Vibrotactile versus mechanotactile sensory feedback," Neural Systems and Rehabilitation Engineering, IEEE Transactions on, vol. 21, no. 1, pp. 112-120, Jan 2013.

[40] J. Bell, S. Bolanski, and M. H. Holmes, "The structure and function of pacinian corpuscles: A review," Progress in Neurobiology, vol. 42, no. 1 , pp. 79-128, 1994.

[41] D. Mahns, N. Perkins, V. Sahai, L. Robinson, and M. Rowe, "Vibrotactile frequency discrimination in human hairy skin," J Neurophysiol, vol. 95, pp. 1442-1450, 2006

[42] S. Lederman, "The perception of surface roughness by active and passive touch," Bulletin of Psychonomic Society, vol. 18, no. 5, pp. 253-255, 1981.

[43] E. P. Scilingo, N. Sgambelluri, D. De Rossi, and A. Bicchi, "Towards a haptic black box for free-hand softness and shape exploration," in Robotics and Automation, 2003. Proceedings. ICRA'03. IEEE International Conference on, vol. 2. IEEE, 2003, pp. 2412-2417.

[44] A. Moscatelli, M. Mezzetti, and F. Lacquaniti, "Modeling psychophysical data at the population-level: The generalized linear mixed model," Journal of Vision, vol. 12, no. 11, pp. 1-17, 2012

[45] B. M. Bolker, M. E. Brooks, C. J. Clark, S. W. Geange, J. R. Poulsen, M. H. H. Stevens, and J.-S. S. White, "Generalized linear mixed models: a practical guide for ecology and evolution," Trends in ecology \& evolution, vol. 24, no. 3, pp. 127-135, 2009. 